\title{
The Perchlorate Reduction Genomic Island: Mechanisms and Pathways of Evolution by Horizontal Gene Transfer
}

\author{
Ryan A. Melnyk ${ }^{1,2}$ and John D. Coates ${ }^{*}$
}

\begin{abstract}
Background: Perchlorate is a widely distributed anion that is toxic to humans, but serves as a valuable electron acceptor for several lineages of bacteria. The ability to utilize perchlorate is conferred by a horizontally transferred piece of DNA called the perchlorate reduction genomic island (PRI).

Methods: We compared genomes of perchlorate reducers using phylogenomics, SNP mapping, and differences in genomic architecture to interrogate the evolutionary history of perchlorate respiration.

Results: Here we report on the PRI of 13 genomes of perchlorate-reducing bacteria from four different classes of Phylum Proteobacteria (the Alpha-, Beta-, Gamma- and Epsilonproteobacteria). Among the different phylogenetic classes, the island varies considerably in genetic content as well as in its putative mechanism and location of integration. However, the islands of the densely sampled genera Azospira and Magnetospirillum have striking nucleotide identity despite divergent genomes, implying horizontal transfer and positive selection within narrow phylogenetic taxa. We also assess the phylogenetic origin of accessory genes in the various incarnations of the island, which can be traced to chromosomal paralogs from phylogenetically similar organisms.

Conclusion: These observations suggest a complex phylogenetic history where the island is rarely transferred at the class level but undergoes frequent and continuous transfer within narrow phylogenetic groups. This restricted transfer is seen directly by the independent integration of near-identical islands within a genus and indirectly due to the acquisition of lineage-specific accessory genes. The genomic reversibility of perchlorate reduction may present a unique equilibrium for a metabolism that confers a competitive advantage only in the presence of an electron acceptor, which although widely distributed, is generally present at low concentrations in nature.
\end{abstract}

\section{Background}

Although widely distributed in the environment, perchlorate $\left(\mathrm{ClO}_{4}^{-}\right)$is generally present at low concentrations ( 14 ng. $\mathrm{L}^{-1}$ across the continental US, Alaska, and Puerto Rico) [1]. While it is toxic to humans, it serves as a valuable electron acceptor for several lineages of bacteria. The canonical microbial metabolism of perchlorate depends on its reduction to chlorite $\left(\mathrm{ClO}_{2}^{-}\right)$in the bacterial periplasm by perchlorate reductase, and the dismutation of chlorite to chloride and molecular oxygen [2]. The biogenic molecular oxygen is not released extracellulary but rather is reduced by the same microorganism, generally through the use of a high-affinity

\footnotetext{
* Correspondence: jdcoates@berkeley.edu

'Department of Plant and Microbial Biology and Energy Biosciences Institute, University of California, Berkeley, CA 94720, USA

Full list of author information is available at the end of the article
}

cytochrome $c b b_{3}$ oxidase. The microaerobic portion of this respiration means that dissimilatory perchloratereducing bacteria (DPRB) uniquely straddle the realms of both aerobic and anaerobic respirations [2]. Genetic studies in the model DPRB Azospira suillum PS have revealed that many of the genes essential for this metabolism are located on a genomic island [3] previously identified in a comparative genomic analysis of four DPRB genomes [4]. This genomic island, designated the perchlorate reduction genomic island (PRI), has clear signatures of horizontal gene transfer (HGT).

Since our original identification of the PRI, we have accumulated 9 additional genome sequences of DPRB, including isolates from the Epsilon- and Gammaproteobacteria [5,6]. Additionally, we have increased the depth of sampling in certain genera, as there are now three sequenced isolates in the Azospira genus, and two in the 
Magnetospirillum genus [7]. In this study, we set out to increase our understanding of the mechanisms of the evolution of PRI. Specifically, we sought to identify mechanisms of integration and excision of the PRI, in addition to uncovering the evolutionary history of the individual genes in the PRI. In answering these questions, we made observations about the evolution of the PRI that led us to propose new explanations for the origin, evolution, and ecological significance of bacterial perchlorate respiration.

\section{Methods}

All genome sequences are publicly available on the IMG database. The accession numbers (or locus tags, for IMG sequences) for all genes discussed in the manuscript can be found in Additional file 1: Table S2. For the genomes sequenced in this study, sequencing methodology and statistical information can be found on the IMG database. Briefly, genomes were sequenced using $150 \mathrm{bp}$ paired end reads on an Illumina HiSeq 2000 and the resulting reads were then assembled using velvet.

\section{MLSA analysis}

Well-known taxa were chosen for illustrative purposes in the Proteobacterial dataset (Fig. 1). We eliminated redundancy at the genus level in the Alpha- and Betaproteobacteria datasets prior to enriching the Rhodospirillaceae and Rhodocyclacease families with draft proteomes from the Joint Genome Institute's IMG database (Figs 2 and 3). These two families are home to all of the perchloratereducing isolates in the Alpha- and Betaproteobacteria classes; thus, we hoped that denser taxon sampling of these families would increase our resolution of relationships among perchlorate-reducing bacteria and also provide insight into the origin of accessory genes in the PRI. In each of the datasets, we included well-established outgroups (e.g. using Firmicutes as outgroups for all Proteobacteria).

To detect orthologs, we used an all-vs-all pairwise BLASTP approach to identify orthologs between each possible pair of proteomes in each dataset encode using inParanoid [8]. Each pairwise inParanoid output was used as input to the QuickParanoid clustering algorithm to identify clusters of homologous proteins (http:// pl.postech.ac.kr/QuickParanoid/). We designated orthologs as those clusters that had exactly one representative from each organism in each dataset (i.e. Proteobacteria, Alphaproteobacteria, Betaproteobacteria, or Rhodocyclaceae). Each set of orthologs was aligned using MUSCLE [9], and the resulting alignments were processed using Gblocks [10] running parameters designed to maximize retention of all informative sites [11]. The alignments were concatenated and converted to Phylip format and

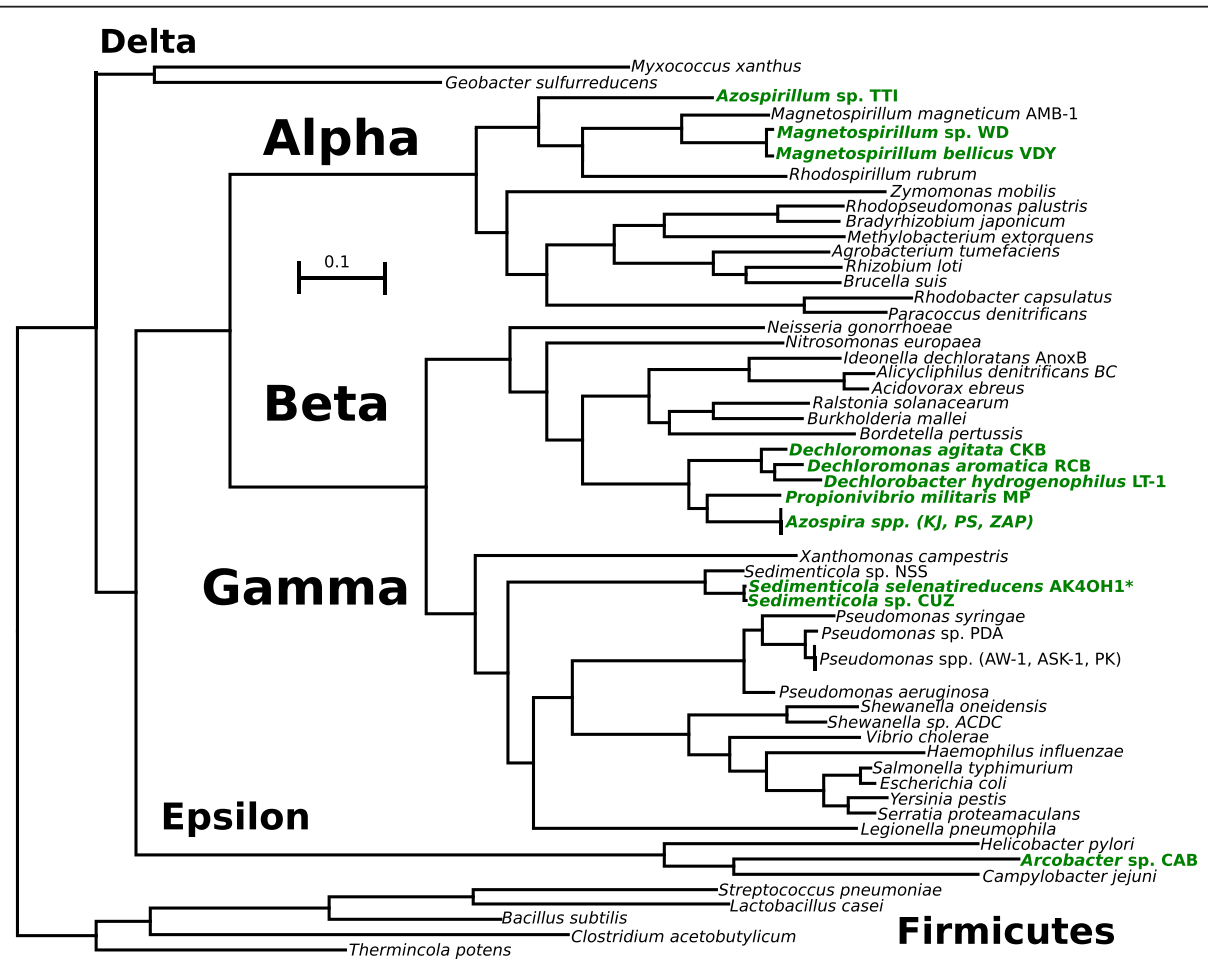

Fig. 1 Phylogeny of the Proteobacteria highlighting DPRB. The dataset used to generate this phylogeny consisted of 90 taxa and 92 conserved orthologous genes, resulting in an alignment of 13066 amino acid positions. Using the LG substitution model, this tree was determined to be the most likely of 100 independently calculated trees, and all nodes were fully supported over 100 bootstrap replicates. DPRB are bolded and in green. The scale bar indicates the branch length that correspond to 0.1 amino acid substitutions per site 


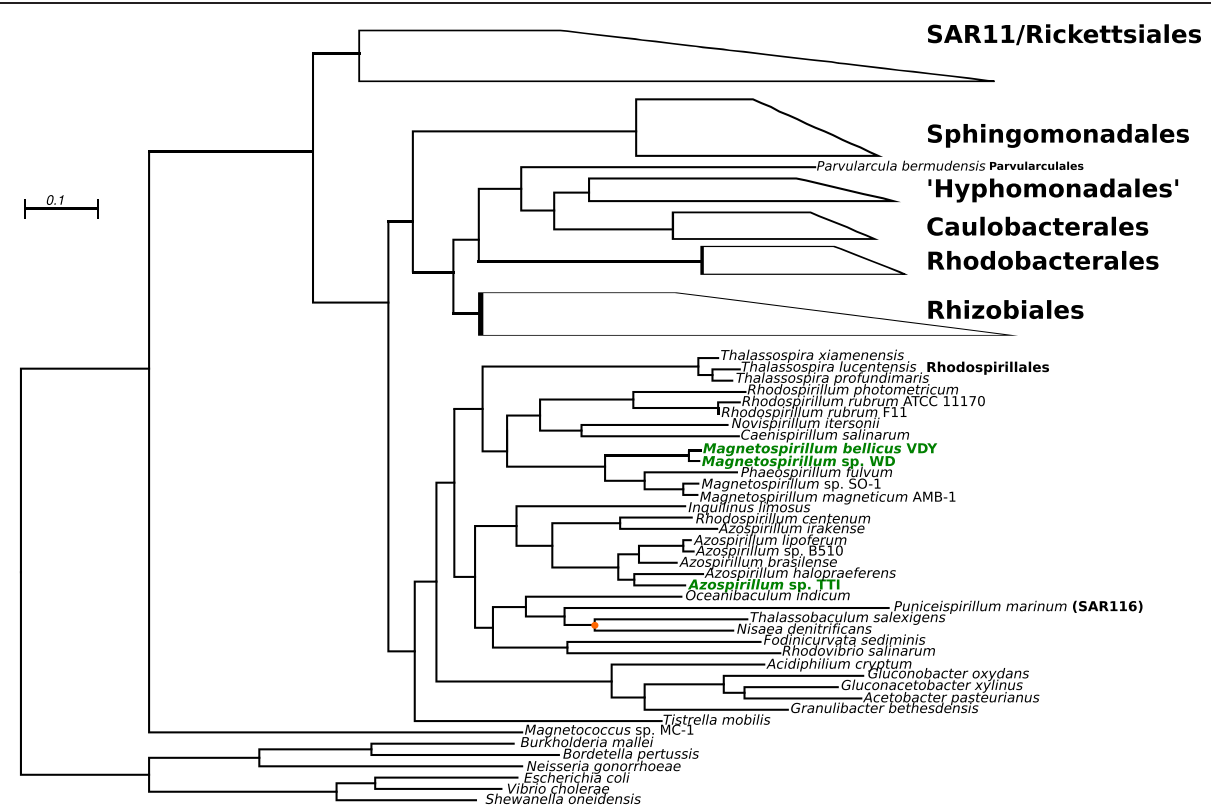

Fig. 2 A tree detailing the relationships within the Alphaproteobacteria, focusing on the Rhodospirillales order. The alignment consisted of 9766 amino acids across 80 genes from 96 taxa and the phylogenetic tree was calculated under the LG substitution matrix using 100 bootstraps and 50 inferences to pick the best tree. All nodes had $100 \%$ bootstrap support with the exception of the node marked with the orange dot. DPRB are bolded and in green. The scale bar indicates the branch length that correspond to 0.1 amino acid substitutions per site

an appropriate model of amino acid substitution was selected using ProtTest 3 [12]. Using the best model and the previously selected outgroup, phylogenetic trees were generated using RAxML [13]. A tree topology was calculated using a maximum-likelihood method for choosing the best tree from a number of independent inferences. A number of bootstrap replicates were performed as well on each dataset, and these were drawn onto the most likely tree to generate support values for each node. This method is described thoroughly in the RAxML manual. The amino acid substitution model and the number of independent inferences and bootstraps are given in the figure legends for each of the four datasets.

In order to simplify the viewing of the Alphaproteobacteria and Betaproteobacteria trees, we collapsed taxonomic groups at the order level based on the published taxonomy. In general, there was robust support for all taxonomic orders with the exception of three species from order Rhodobacterales in the Alphaproteobacteria (Hirschia baltica, Hyphomonas neptunium, and Maricaulis maris), which claded with the Caulobacterales. This was previously observed for the genome of the species Hyphomonas neptunium, which was assigned to the Rhodobacterales based on $16 \mathrm{~S}$ phylogeny, but groups with the Caulobacterales on the basis of housekeeping gene sequences [14]. These three species are all members of family Hyphomonadaceae, which we have depicted in our tree as the order-level classification 'Hyphomonadales' following a previously set precedent
[15]. We note that this is currently not an official taxonomic grouping, but our analysis suggests that these species should reside in a sister group to the Caulobacterales or perhaps within that order itself.

The program Mauve was used to generate wholegenome alignments of the Azospira draft genomes (ZAP, KJ, and 6a3) to the finished reference genome of Azospira suillum PS, in addition to aligning contigs from the two Magnetospirillum draft assemblies (VDY and WD).

\section{Individual gene phylogenies}

For the phylogenies of independent genes, we first used a HMMER3 protein similarity search to identify similar proteins to the query of interest in our entire HAMAP/ IMG dataset [16]. We then took all similar proteins and clustered them with a $90 \%$ identity threshold using CDHIT to reduce the complexity of the dataset [17], prior to generating an alignment as before, using MUSCLE and Gblocks. We then calculated a best maximumlikelihood tree from 10 independent inferences using RAxML [13]. We then viewed the tree using Dendroscope [18], and chose an ingroup containing the sequences from the PRI and an appropriate outgroup. The selected sequences were aligned and curated again as before, using MUSCLE and Gblocks. A best amino acid model was determined using ProtTest 3 and a phylogenetic tree with support values was calculated 

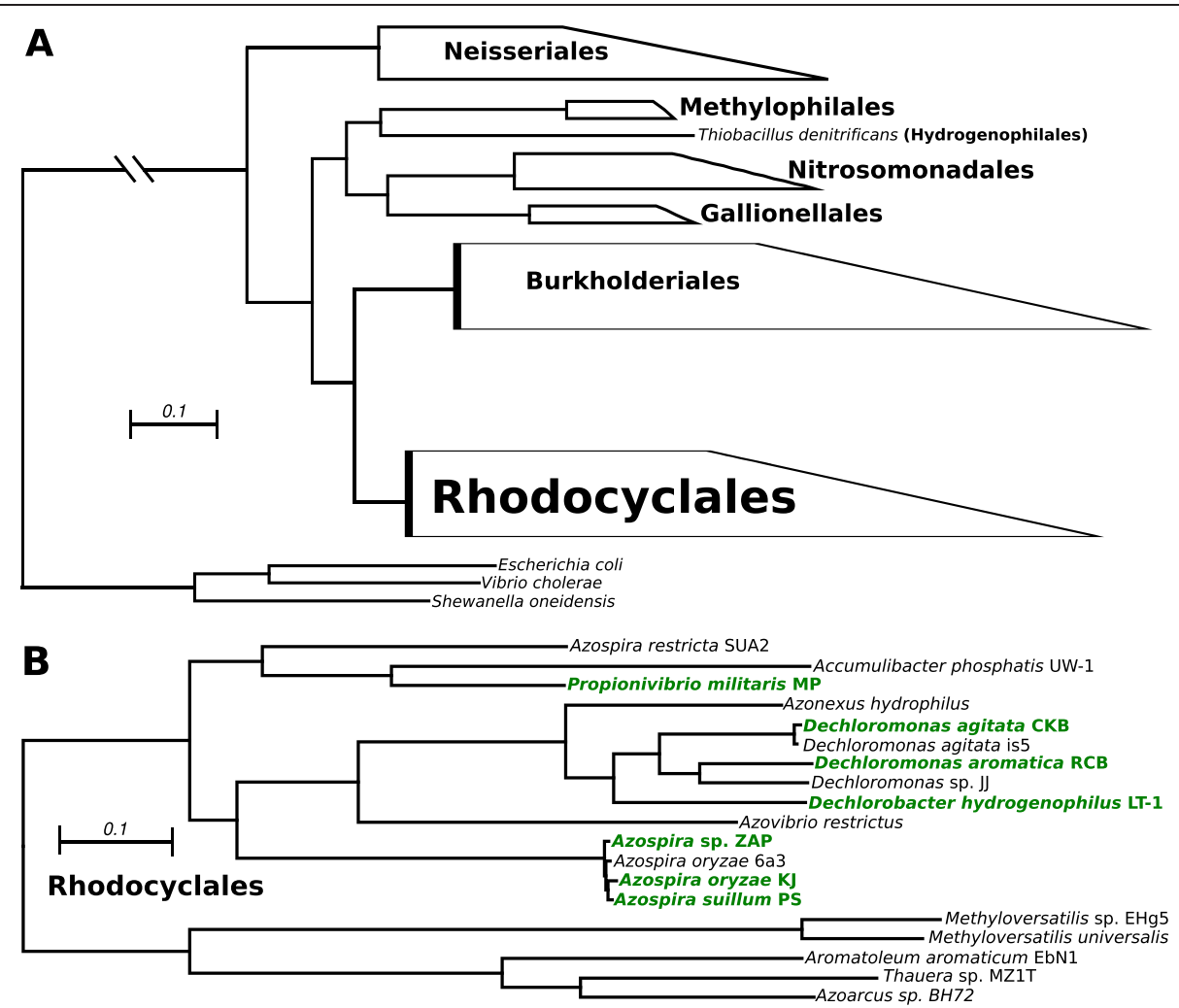

Fig. 3 Phylogenetic trees depicting the Betaproteobacteria class and the Rhodocyclales, the only DPRB-containing order. a The alignment consisted of 21601 non-redundant amino acid positions from 147 orthologous genes. The tree was calculated using the LG substitution matrix and overlaying the results of 100 bootstrap replicates over the most likely tree of 50 independent inferences. $\mathbf{b}$ The Rhodocyclales subtree is rooted to taxa from a sister clade within the same order, which were chosen based on their position in the tree made for all Betaproteobacteria (i.e. Fig. 5A). The alignment used to construct this tree consisted of 139587 amino acid positions from 229 genes across 19 taxa. The tree was calculated under the LG +F substitution matrix and was generated by drawing 100 bootstrap replicates on the best tree chosen from 50 independent maximum-likelihood inferences. For both trees, all nodes had $100 \%$ bootstrap support and the scale bar indicates the branch lench corresponding to 0.1 substitutions per site. DPRB are bolded and in green

as before using RAxML and the best tree/bootstrap method. In several instances, RogueNaRok (https:// github.com/aberer/RogueNaRok) was used to remove rogue taxa that led to conflicting bootstrap topologies and thus ambiguous phylogenetic signals, prior to tree recalculation.

\section{Availability of Supporting Data}

The data sets supporting the results of this article are included within the article and its additional files. All genome sequences have been uploaded to the IMG database and will be made publicly available upon acceptance of the manuscript. The accession numbers (or locus tags, for IMG sequences) for all genes discussed in the manuscript can be found in Additional file 1: Table S2.

\section{Ethics}

This work did not require humans, human data, or animals, thus a statement of ethics is not required.

\section{Results}

Perchlorate/chlorate reducers are restricted to certain subclades within the Proteobacteria

All of the genomes used in this study are publically available and their accession numbers along with associated references for their phenotypic descriptions are provided in Additional file 2: Table S1. To assess the phylogenetic relationships between DPRB and other bacteria, we adopted a multi-locus sequence analysis (MLSA) method that relies upon an in-house pipeline for identifying as many orthologs as possible within a given group of bacteria. While MLSA methods do not fully recapitulate the reticulate and piecewise nature of bacterial genome evolution and speciation [19], they are generally satisfactory for resolving a consensus pattern of species divergence [20], particularly when many "taxonomically complete" loci are used [21].

Although DPRB isolated to date are scattered throughout the Proteobacteria (Fig. 1), their presence within classes seems to be restricted. In the Alphaproteobacteria, 
DPRB are only found in the family Rhodospirillaceae (Fig. 2), while in the Betaproteobacteria, they are present in only one subgroup within the Rhodocyclaceae (Fig. 3). This has been observed since the advent of molecular phylogeny in the context of DPRB [2], but our phylogenetic analysis confirms and strengthens these inferences by making use of the entire genome, rather than the $16 \mathrm{~S}$ genes of DPRB isolates [22, 23]. DPRB isolates from the Epsilonproteobacteria and Gammaproteobacteria have only recently been characterized and sequenced $[5,6]$, thus it is impossible to make sense of their distribution within their respective taxonomic classes until more strains have been isolated.

\section{PRIs have a diverse, yet functionally similar, 'core' set of genes}

The original identification of the PRI consisted of 4 separate island 'cores', which were defined by beginning at the pcrA and cld genes and using pairwise BLAST analysis to detect genes shared by more than one DPRB [4]. With the sequencing of additional PRIs, more classes of accessory genes have been observed, although they tend to fit within four main functional groups: transcriptional regulation, electron transport, oxidative stress resistance, and molybdenum cofactor biogenesis (Fig. 4).

Transcriptional regulation in response to environmental flux was identified as a possible role for genes in the PRI, specifically via a histidine kinase signaling pathway [4]. This was confirmed using genetics, as pcrPSR are each independently required for perchlorate reduction in A. suillum PS [3]. The pcrSR genes in Propionivibrio militaris MP [22], Dechloromonas aromatica RCB [24], and Azospira spp. form a monophyletic group with the pcrSR genes from Magnetospirillum spp. PRIs. The orientation of $p c r S$ and $p c r R$ on different coding strands is conserved in this group, but Sedimenticola spp. and Dechlorobacter hydrogenophilus LT-1 each have histidine kinase systems with unique genomic and domain organization (Fig. 4). Nevertheless, we have used the pcrPSR nomenclature developed in PS for these organisms to illustrate that they may play a similar role in transcriptional regulation. In the Alphaproteobacteria DPRB, there is the possibility of other transcriptional regulators involved with perchlorate reduction as the PRI of Azospirillum sp. TTI contains a crp-type regulator and the PRI of Magnetospirillum spp. contain a lysRtype regulator (Fig. 4).

The exact mechanism of electron transport from the inner membrane to the periplasmic perchlorate reductase is unknown. PcrC is a soluble tetraheme cytochrome previously thought to be an important part of perchlorate reductase function [25]. The pcrC gene is essential for perchlorate reduction in A. suillum PS [3], although its complete absence in the genome of Arcobacter sp. $\mathrm{CAB}$ indicates that it is not universally required for perchlorate reductase function [5]. The electron transport mechanism is difficult to infer from genomic observation alone, as PRIs have many genes that could be involved in transporting reducing equivalents (red highlighted genes, Fig. 4). Two genes occur in multiple PRIs: pcrQ, which encodes a quinol dehydrogenase tetraheme cytochrome similar to well-characterized proteins such as CymA, $\mathrm{NrfH}$, and NapC [26], and pcrO, which is homologous to the gamma subunit of ethylbenzene dehydrogenase [27].

Oxidative stress defense has recently emerged as an important fitness determinant during (per)chlorate reduction [28], likely due to the inadvertent accumulation of the side product hypochlorite from chlorite dismutation [29]. The key genes in this functional group are the

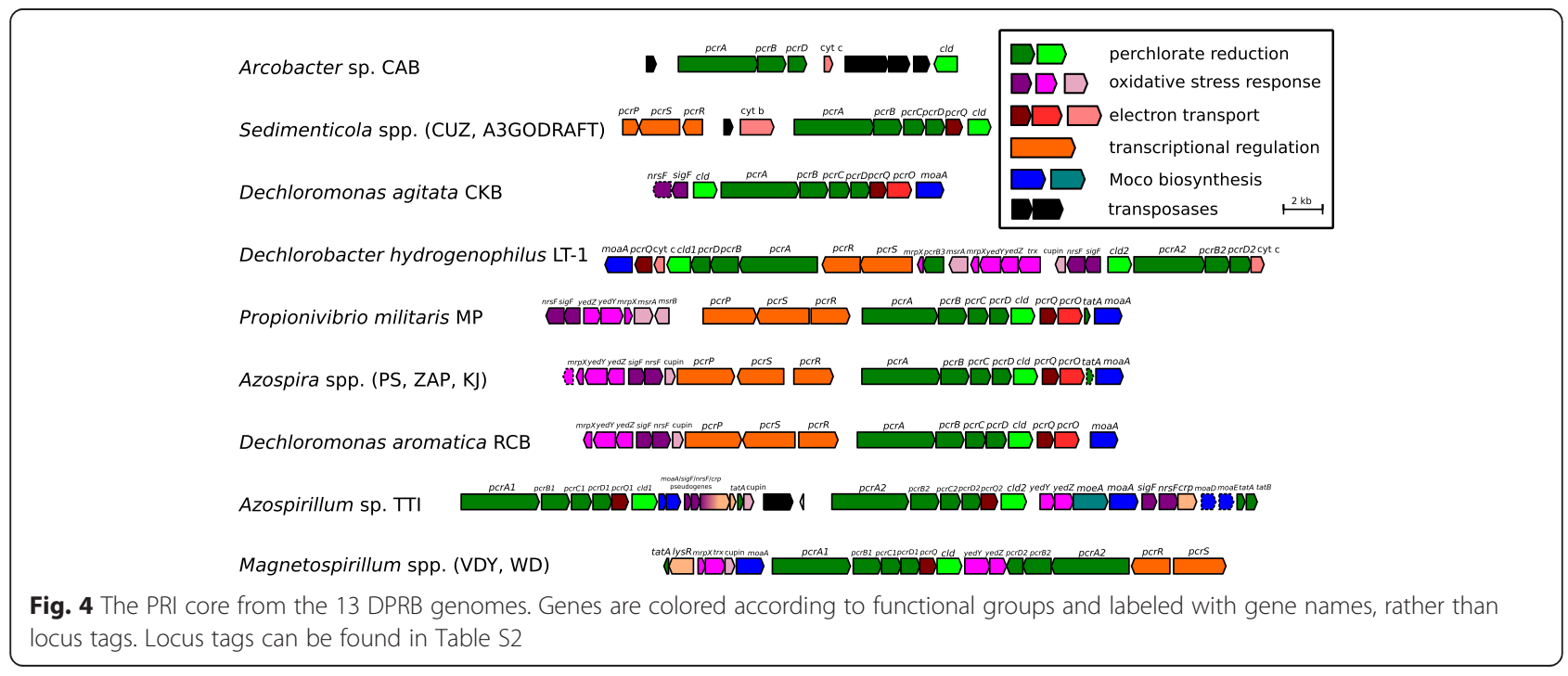


sigF/nrsF sigma factor/anti-sigma factor system, the putative methionine sulfoxide reductase genes yed $Y Z$, and the methionine-rich peptide gene $m r p X$, which form a periplasmic methionine-cycling mechanism to detoxify hypochlorite [30]. New PRIs have reiterated the importance of this function, as $P$. militaris $\mathrm{MP}$ and $D$. hydrogenophilus LT-1 also contain homologs of the methionine sulfoxide reductase gene $m s r A$ and $m s r B$ (Fig. 4). However, unlike the cytoplasmic housekeeping version of these genes, they are predicted to be exported to the periplasm, where the bulk of perchlorate-associated oxidative stress is likely to occur.

Perchlorate reductase is part of the diverse DMSO reductase protein family and thus contains a molybdenum ion bound by two pterin guanine dinucleotide groups, also known as the molybdenum cofactor, or Moco [31, 32]. Within the PRI of every DPRB in the Alpha- and Betaproteobacteria, there is a $\operatorname{moaA}$ homolog (Fig. 4). MoaA is a radical SAM-dependent enzyme which catalyzes the first step in pterin synthesis from GTP [31, 33]. All of the known DPRB isolates contain a homolog of moaA elsewhere in the genome, however, the PRI moaA gene appears to be important for perchlorate reduction in minimal media at least in the case of A. suillum PS [3].

\section{PRIs are incorporated at distinct genomic sites, even within a family}

The exact boundaries of the PRI outside of the core were not delineated in its initial observation, but genes associated with mobilization were found outside of the core [4]. Variability in mobilization genes was even observed between PRIs with similar cores, suggesting that the core genes can be found in various types of mobile elements [4]. In the complete genome of the canonical DPRB, A. suillum PS, the PRI core genes are located close to a proline tRNA gene [4]. Upon closer inspection, this gene includes a 48-bp sequence that is also found at the other end of the PRI (Fig. 5). Using this region as a scaffold, we were able to assemble a contig with the same genomic organization in the other two Azospira DPRB strains, KJ and ZAP [34]. We noticed that the genes outside of these repeat regions were
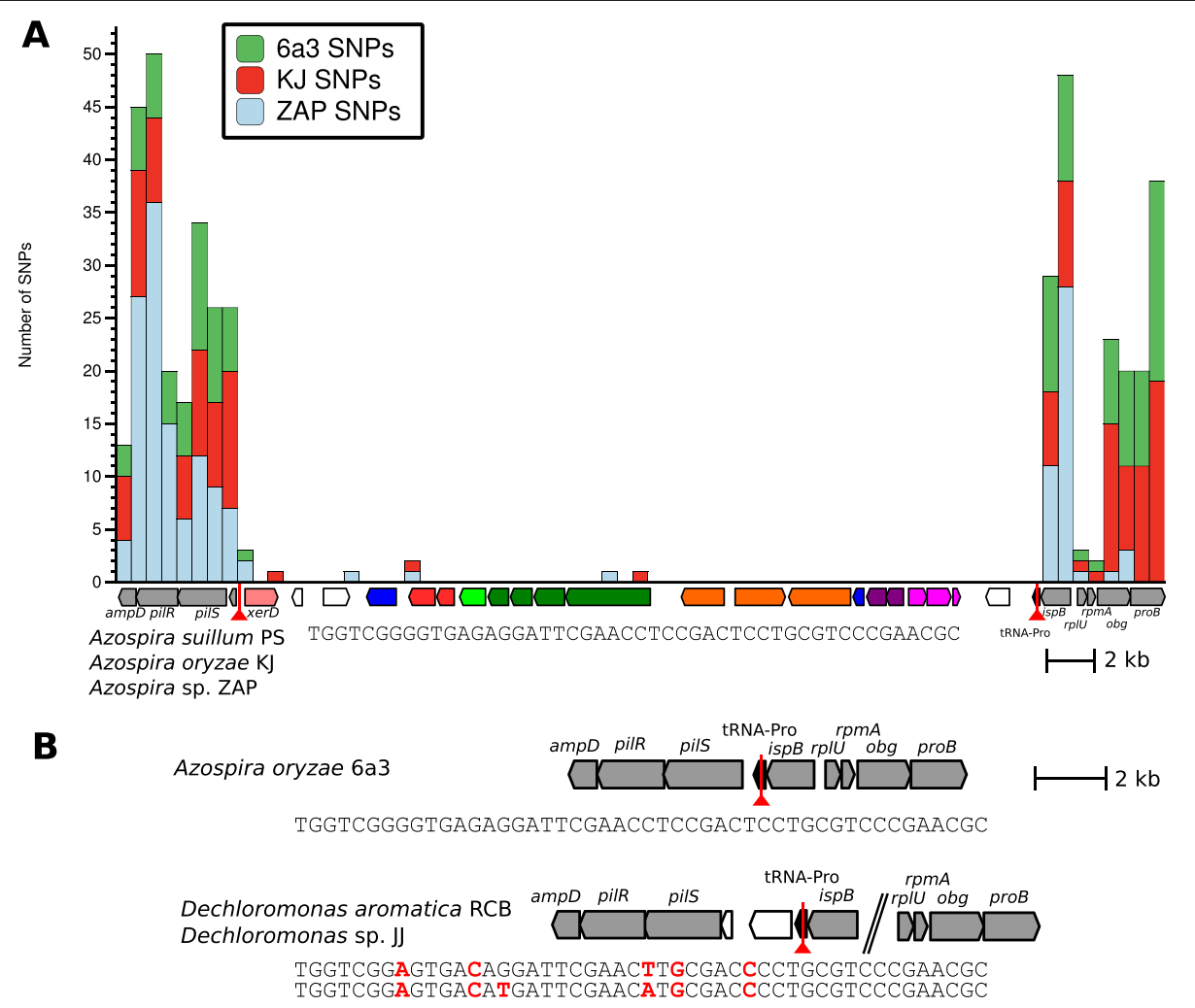

Fig. 5 Diagram of the PRI and flanking regions in Azospira spp. a A plot of the SNP abundance of Azospira spp. 6a3, KJ, and ZAP relative to the finished PS genome seqence. Because $6 a 3$ lacks the PRI, SNPs were counted over a 500-bp window and overlaid on the PRI and flanking regions from PS, as individual columns in the histogram. The nucleotide sequence of the 48-bp site of insertion in the proline tRNA is located below the gene diagram. $\mathbf{b}$ This image contains the flanking regions in the non-DPRB A. oryzae $6 a 3$ and two Dechloromonas spp. demonstrating the synteny of the flanking regions prior to insertion of the PRI. Here, the nucleotide sequence of the putative site of integration within the proline tRNA is shown for all strains (indicated by the red line on the genetic diagrams). $6 a 3$ has an identical insertion site to the DPRB Azospira spp., but the Dechloromonas spp. have several SNPs 
orthologs detected in all Rhodocyclaceae (e.g. ispB, pilSR) so we looked at their organization in other strains. These flanking genes frequently form a contiguous region in both other DPRB and non-perchlorate reducing strains such as Azospira oryzae 6a3, confirming that the PRI was integrated into the proline tRNA (Fig. 5). In other Rhodocyclaceae, however, this operon is not intact but has had a different genomic island incorporated, suggesting that this specific tRNA is a frequent target of mobile elements. Outside of the PRI core but within the boundary formed by the direct repeats is a site-specific recombinase homologous to $x e r D[35,36]$, thus providing a possible mechanism for PRI integration.

The boundaries of the entire PRI were unclear in the genome of $D$. aromatica $\mathrm{RCB}$, which lacked homologs of the non-core genes from the Azospira spp. PRI. However, using our ortholog detection approach to compare the genome of strain RCB to its close relative, Dechloromonas sp. JJ, we were able to delineate the exact boundaries of the PRI in RCB. The RCB PRI is much larger, spanning $145 \mathrm{kB}$ and containing genes for plasmid replication and conjugation. This structure denotes an integrative and conjugative element, or ICE, and may be capable of catalyzing its own excision, transmission and perhaps replication [37].

Due to the fragmentary nature of draft genome assemblies, we were unable to confidently map the location of the PRI insertion in P. militaris MP or D. agitata CKB. In D. hydrogenophilus LT-1, the PRI is inserted in a region with conserved synteny in other Rhodocyclaceae. However, there are no obvious 'scars' or evidence for a mechanism of integration and no close non-perchlorate reducing relative to $\mathrm{LT}-1$, so the history of its PRI is unclear. The $D$. agitata CKB PRI is located on its own relatively small contig, but re-mapping of paired-end reads to the contigs using bowtie indicates that it is flanked by near-identical insertion sequences, thus forming a composite transposon. This organization was seen in chlorate-reducing bacteria, where the genetic 'cargo' for the metabolism is located between two insertion sequences, which enable its mobilization [38].

The PRIs from several isolates within the genera Azospira and Magnetospirillum are the result of recent HGT and positive selection

The conservation of the PRI in the three DPRB of the four sequenced Azospira species and its integration into an identical location in all three (Fig. 5), might be suggestive of one acquisition event prior to the divergence of the four strains, followed by loss of the PRI in strain 6a3. However, the nucleotide comparisons between the Azospira strains do not support such a history. By mapping SNPs in the three Azospira draft sequences (strains $\mathrm{KJ}$, ZAP, and 6a3) to the reference strain PS genome, we see that the PRI in KJ and ZAP has very few SNPs, especially when compared to the conserved genes outside the PRI, which have mutation rates roughly average for what is expected from the genome as a whole (Fig. 5, Table 1). The lack of nucleotide divergence between the three PRIs, even at synonymous sites and intergenic regions indicates that the PRI was acquired independently and recently by the three extant Azospira DPRB isolates. Of note is that two of the six SNPs in the PRI result in amino acid substitutions in the sequence of PcrA, the initial reductase in the perchlorate reduction pathway (I431L in Azospira sp. ZAP, Y736H in Azospira sp. KJ), raising the possibility that parts of the PRI may be evolving under positive selection.

\section{PcrAB and Cld: Monophyletic anchors of an ancestral PRI}

Although the $\operatorname{pcr} A B$ and cld genes found in perchloratereducing bacteria have phylogenies suggestive of horizontal gene transfer, they both are largely monophyletic and thus support a single ancestral PRI (Figs 6 and 7). The only exception to this is the co-option of the cld gene sequence in the formation of chlorate-reduction transposons (e.g. in Ideonella dechloratans and Alicycliphilus denitrificans); however, these cld alleles likely came from the PRI of an existing DPRB [38]. We rooted the Cld sequences from DPRB and two chlorate-reducing bacteria by using a metagenomic sequence and the Cld sequence from 'Candidatus Nitrospira defluvii' [38, 39]. A more comprehensive phylogeny of Cld sequences indicates that these sequences are intermediates between DPRB Clds and the broadly distributed cytoplasmic Cld [38, 40]. The PcrAB sequences were concatenated and used to construct an alignment rooted to three sequences of Archaeal periplasmic nitrate reductases [41, 42], which are closely related to extant PcrAB sequences [38].

\section{Discussion}

Why only certain clades?

Although perchlorate reduction is distributed broadly among four different classes within the Proteobacteria,

Table 1 SNP frequency in the PRI and genomes of Azospira spp.

\begin{tabular}{lllll}
\hline & PRI SNPS & genome SNPs & PRI frequency & genome frequency \\
\hline KJ vs. PS & 3 & 74295 & $0.01 \%$ & $1.97 \%$ \\
ZAP vs. PS & 3 & 66033 & $0.01 \%$ & $1.75 \%$ \\
6a3 vs. PS & n/a & 53194 & n/a & $1.41 \%$ \\
\hline
\end{tabular}




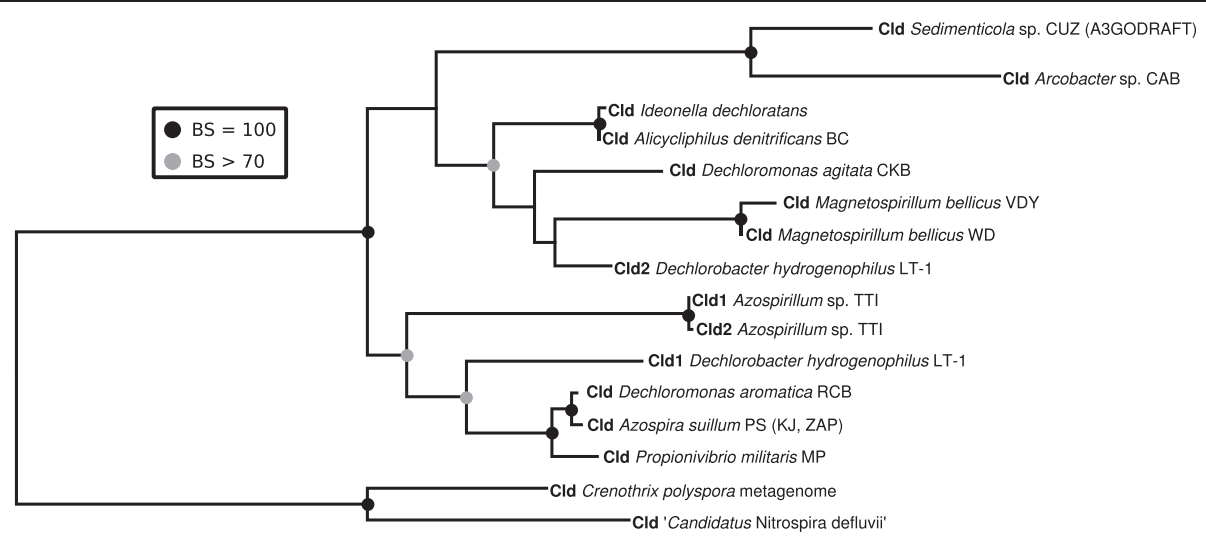

Fig. 6 Phylogenetic tree of Cld sequences. Phylogenetic tree constructed using an alignment of Cld sequences from DPRB and rooted to two sequences predicted to be periplasmic Cld sequences not found in DPRB. The alignment used to construct this tree had 255 amino acid positions. The best tree was calculated using the WAG substitution model and was chosen from 200 independent inferences, and bootstrap support values were calculated from 1000 bootstrap replicates

the taxonomic scope of isolates within those classes is narrow. The reason for this is unknown, but is plausibly the result of the interplay of multivariate factors. First, it is very likely that isolation and enrichment techniques are biased for a certain type of DPRB, likely a copiotroph that has a fast growth rate. It may be that these organisms represent only one of several microbial ecological strategies that make use of perchlorate reduction. Indeed, it has previously been observed that altering traditional enrichment parameters such as $\mathrm{pH}$, temperature, or salinity is sufficient to alter the perchlorate reducers isolated [5, 22]. Additionally, $16 \mathrm{~S}$ or metagenomicsbased analyses of perchlorate-reducing communities may uncover a taxonomically richer set of organisms than those currently present in DPRB isolate collections; this avenue has recently been taken by several research groups studying perchlorate-removing bioreactor communities [43, 44].

An organism may also require additional traits in order to become a successful perchlorate-reducer. Generally speaking, perchlorate reducers are motile aquatic organisms, can utilize a variety of electron donors (and acceptors), and are generally non-fermentative facultative anaerobes [2]. It may be that only certain taxonomic groups such as Rhodocyclaceae and Rhodospirillaceae are prepared to be successful in environments such as these, and thus these groups harbor the most cosmopolitan DPRB.

Another possibility is that this taxonomic restriction is enforced by the mechanism of PRI integration. The Azospira PRI is integrated at a very specific site in a tRNA, and the exact sequence of the site is not conserved in other Rhodocyclaceae. This specificity may mean that Azospira has an advantage in certain environments, where it can acquire a version of the PRI easily through site-specific integration at a neutral genomic location. Furthermore, our phylogenetic analysis indicates that accessory genes from the PRI are often related to genes found in the host organism. We hypothesize that this streamlines PRI integration with

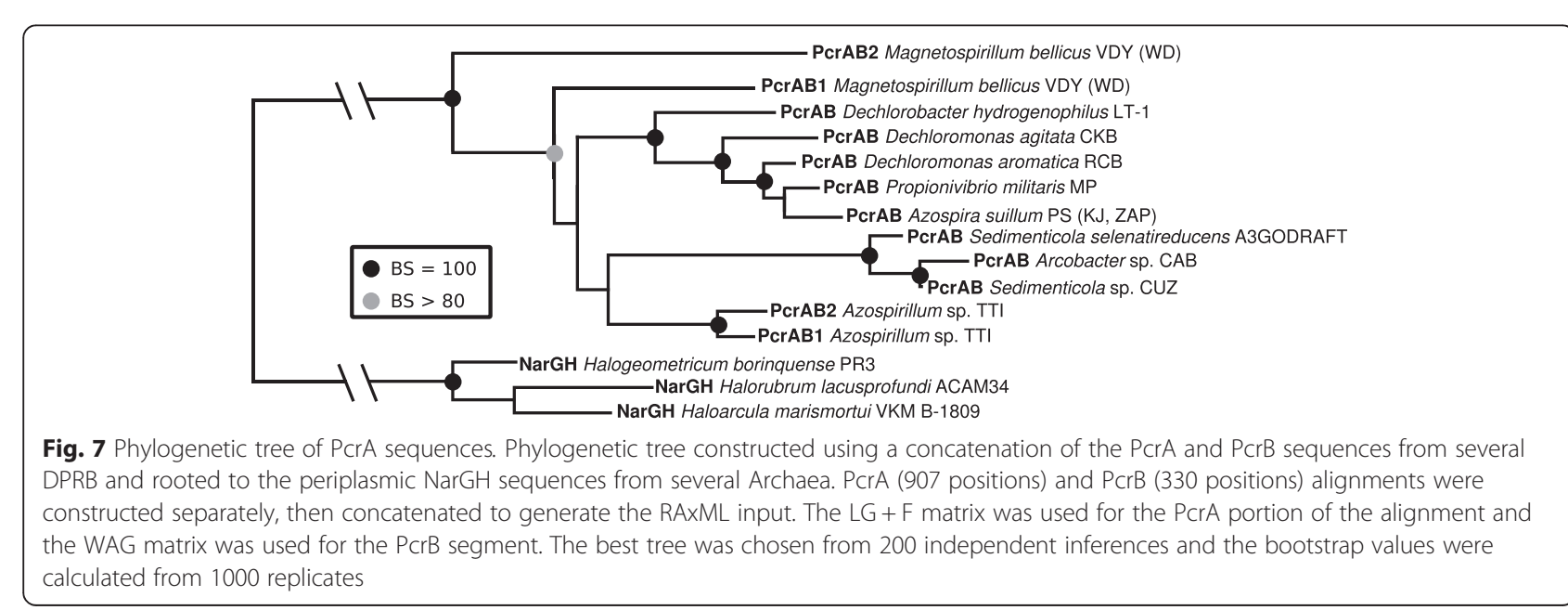


host metabolism, specifically in the realms of regulation and electron transport. Specificity of both integration mechanisms and accessory genes results in a positive feedback loop where a PRI becomes more and more optimized for a given lineage. This may in practice reduce DPRB diversity, as an Azospira strain that has acquired a PRI tailored for its needs will be much more competitive than an organism attempting to piece together a PRI de novo.

\section{Why is perchlorate reduction so obviously associated with horizontal gene transfer?}

The lack of the island in A. oryzae sp. $6 \mathrm{a} 3$ and the nucleotide identity shared in the PRIs of perchloratereducing Azospira spp. indicates that perchlorate reduction is not a conserved function of this genus, nor is it for any taxonomic group we have studied. Additionally, the ubiquitous presence of HGT "scars" associated with perchlorate reduction indicates that it is frequently propagated via horizontal gene transfer. This stands in stark contrast to reductive pathways such as denitrification, which are present in many different Proteobacteria and vary little in operon organization across broad phylogenetic groups.

The evolutionary trajectories of these two disparate metabolisms are likely shaped by the abundance of the respective electron acceptors in the environment. Denitrification is ubiquitous; nitrate is found in many types of aquatic and terrestrial environments at moderate concentrations and is continuously generated from inorganic nitrogen sources by nitrifying bacteria. Conversely, the are no known biological neogenesis mechanisms for perchlorate and it is rarely found in ecologically relevant quantities due to its slow abiotic generation in the atmosphere. The discrepancy between the abundance of these two compounds mimics the discrepancy in the frequency of the respective pathways in isolated bacteria.

This "rare perchlorate" hypothesis implicitly states that maintaining the PRI in the absence of perchlorate has a fitness cost. Although perchlorate is much more limited than nitrate in the environment, there are clearly situations where it is adaptive to have the ability to reduce perchlorate. Perchlorate accumulates in dry environments such as the Atacama Desert and the Antarctic Dry Valleys [45], but is also deposited across much wider geographic scales [1]. However, because its concentration in groundwater is tied to stochastic rainfall and irrigation events, many geographical areas with high washout may never reach a biologically relevant concentration of perchlorate, particularly prior to industrial contamination of perchlorate. These factors result in non-overlapping niches, or microenvironments where DPRB are successful but may be geographically isolated from other similar microenvironments, thus limiting their dispersion. In other words, we hypothesize that the frequency of environments where DPRB are successful is low enough or that selection against the PRI in nonperchlorate environments is negative enough, that the PRI is never fixed into a taxonomic group.

The stochastic pattern of natural perchlorate deposition and lack of continuous positive selection provides a plausible hypothesis for why an ancient metabolism would exist in such a low equilibrium abundance among bacterial genomes, yet continue to persist. However, release of anthropogenic perchlorate into the environment may be changing this equilibrium, providing a continuously selective environment for DPRB or increasing the abundance of PRIs globally. The minute number of SNPs among PRIs from the Azospira species relative to the rest of the respective genomes indicates a very recent acquisition of the PRI, especially relative to the divergence of the genomes as a whole. Further sequencing of genomes and PRIs of isolates and populations from contaminated sites, bioreactors, and pristine dry environments will be essential in gaining a more complete understanding of the dynamics of PRI evolution.

\section{Ancient evolution of perchlorate reduction}

All extant sequences of PcrA and Cld are monophyletic and are linked together on the same "piece" of DNA in canonical DPRB. This suggests that all modern PRIs descended from a single progenitor PRI. Hypothetically, this progenitor PRI required three critical steps in its assembly. First and most important is the evolution of robust perchlorate reduction activity. Once an organism gains the ability to reduce perchlorate, the problem of reactive chlorine species presents itself. The chlorite dismutase enzymatic function likely predates the linkage of $c l d$ and pcrA, as there are Cld sequences basal to the Cld sequences found in DPRB. However, Cld sequences from DPRB are unique in the sense that they all contain a signal sequence indicating export to the periplasm. We hypothesize that this was another crucial step in the evolution of perchlorate reduction, which allowed chlorite to be dismutated near the site of its production in the periplasm. Once an organism acquired both of these abilities, it became the first perchlorate reducer. However, until these genes became genetically linked, this trait was likely inherited only vertically. The first PRI arose when $c l d$ and pcrA were incorporated on the same piece of DNA that could be transferred between unrelated organisms.

We propose that successful events of PRI horizontal gene transfer that lead to propagation within a new taxonomic group are rare. We also propose that less complex PRIs have a much better chance of being incorporated in taxonomically novel backgrounds; the acquisition of lineage-specific accessory genes comes later, once the PRI is being frequently transferred among 
phylogenetically similar organisms. An example of the early stages of this progress may be the PRIs in Sedimenticola spp. and Arcobacter sp. CAB which both contain transposase 'scars' within the PRI core (Fig. 4).

\section{Recent evolution of perchlorate reduction}

Although extant lineages of perchlorate reduction derive from infrequent HGT events, our genomic data indicates that within these lineages recombination and transfer of the PRI is rampant. The most striking instance of this is the near-identity among the PRIs of the Azospira spp.

The fact that some of the accessory genes are pseudogenes also demonstrates the ongoing evolution of the PRI core (genes with dashed outlines, Fig. 4). Inactivation of genes by altering the start codon or introducing a nonsense mutation is an early step in this process, eventually followed by gene loss [46]. This likely occurs because genes in the PRI served an adaptive purpose in the donor organism, but are either neutral or deleterious in their current host [47].

\section{Conclusions}

Perchlorate reduction is a unique metabolism that combines elements of aerobic and anaerobic respiration. The genomic basis for perchlorate reduction, the PRI, is a unique genomic island as well, encoding complex systems for regulation and oxidative stress response. We have shown in this study that the PRI is primarily associated with horizontal gene transfer on varying degrees of time scales. Understanding the dynamics of horizontal transfer of the PRI, as well as how it becomes integrated into host physiology, will be essential for understanding the ecology of this metabolism and where in the environment these genes exist. Towards this goal, we have integrated sequencing of taxonomically diverse isolates and genetic studies of a model system in order to understand the function of the genes in the PRI. We believe that perchlorate reduction provides an interesting model system for those looking at the intersection of microbial physiology and ecology, and how genomic organization and horizontally transferred genes shape microbial lifestyles and evolution.

\section{Additional files}

Additional file 1: Table S2. List of locus tags for all of the genes mentioned explicitly or present in figures in this manuscript. (XLSX 53 kb)

Additional file 2: Table S1. Information on all 13 genomes used in this manuscript. (XLSX $42 \mathrm{~kb}$ )

\section{Abbreviations}

BLAST: Basic Local Alignment Search Tool; Cld: Chlorite dismutase; Contig: Contiguous region; Crp: Cyclic AMP receptor protein; CymA: Tetraheme cytochrome c; DMSO: Dimethyl sulfoxide; DPRB: Dissimilatory perchlorate-reducing bacteria; Fe-S clusters: Iron sulfur clusters; HGT: Horizontal gene transfer; ICE: Integrative and conjugative element; IspB: Octaprenyl diphosphate synthase; LysR: Lysine biosynthesis transcription regulator; MLSA: Multi-locus sequence analysis;

MoaA: Molybdopterin biosynthesis protein A; Moco: Molybdenum cofactor; Mrp: Methionine rich peptide; Nap: Periplasmic nitrate reductase; Nrf: Periplasmic nitrite reductase; NrsF: Anti-sigma factor; Pcr: Perchlorate reductase; PilR: Two component histidine kinase response regulator; PilS: Two component histidine kinase sensor; PRI: Perchlorate reduction genomic island; SigF: Sigma factor; SNP: Single nucleotide polymorphism; XerD: Site specific tyrosine recombinase; YedYZ: Methionine sulfoxide reductase.

\section{Competing interests}

The authors declare that they have no competing interests.

\section{Authors' contributions}

RAM and JDC conceived of the project. RAM performed all computational work. RAM and JDC developed the evolutionary hypotheses detailed in the discussion and wrote the manuscript. JDC directed the project, cointerpreted the data, and co-wrote the manuscript. RAM performed all bench work, applied all bioinformatics methods, analyzed and co-interpreted data, and co-wrote the manuscript. Both authors read and approved the final manuscript.

\section{Acknowledgements}

Funding for perchlorate research in the lab of J.D.C. comes from the Energy Biosciences Institute. This work used the Vincent J. Coates Genomic Sequencing Laboratory at UC Berkeley, supported by NIH S10 Instrumentation Grants S10RR029668 and S10RR027303. The majority of the computational work described here was carried out using the UC Berkeley Computational Genomics Resource Laboratory under the direction of Ravi Alla, who we would like to acknowledge. We would like to thank lain Clark for invaluable discussions on computational genomics and sharing his incredibly useful python gene plotting script, among other tools. Finally, the rest of the Coates Lab must be acknowledged for many discussions about perchlorate reduction, from the level of pure culture genetics to evolutionary ecology.

\section{Author details}

'Department of Plant and Microbial Biology and Energy Biosciences Institute, University of California, Berkeley, CA 94720, USA. ${ }^{2}$ Physical Biosciences Division, Lawrence Berkeley National Laboratory, Berkeley, CA, USA.

Received: 5 March 2015 Accepted: 3 October 2015

Published online: 26 October 2015

\section{References}

1. Rajagopalan S, Anderson T, Cox S, Harvey G, Cheng Q, Jackson WA. Perchlorate in wet deposition across North America. Environ Sci Technol. 2009:43:616-22.

2. Coates JD, Achenbach LA. Microbial perchlorate reduction: rocket-fueled metabolism. Nat Rev Microbiol. 2004;2:569-80.

3. Melnyk RA, Clark IC, Liao A, Coates JD. Transposon and deletion mutagenesis of genes involved in perchlorate reduction in Azospira suillum PS. mBio. 2014:5:e00769-13.

4. Melnyk RA, Engelbrektson A, Clark IC, Carlson HK, Byrne-Bailey K, Coates JD. Identification of a perchlorate reduction genomic island with novel regulatory and metabolic genes. Appl Environ Microbiol. 2011;77:7401-4.

5. Carlström Cl, Wang O, Melnyk RA, Bauer S, Lee J, Engelbrektson A, et al. Physiological and genetic description of dissimilatory perchlorate reduction by the novel marine bacterium Arcobacter sp. strain CAB. mBio. 2013;4:e00217-13.

6. Carlström Cl, Loutey DE, Wang O, Engelbrektson A, Clark I, Lucas LN, et al. Phenotypic and genotypic description of Sedimenticola selenatireducens strain CUZ, a marine (per)chlorate respiring Gammaproteobacterium and its close relative the chlorate respiring Sedimenticola strain NSS. Appl Environ Microbiol. 2015:81(8):2717-26.

7. Thrash JC, Ahmadi S, Torok T, Coates JD. Magnetospirillum bellicus sp. nov., a novel dissimilatory perchlorate-reducing alphaproteobacterium isolated from a bioelectrical reactor. Appl Environ Microbiol. 2010;76:4730-7. 
8. Remm M, Storm CE, Sonnhammer EL. Automatic clustering of orthologs and in-paralogs from pairwise species comparisons. J Mol Biol. 2001;314:1041-52.

9. Edgar R. MUSCLE: a multiple sequence alignment method with reduced time and space complexity. BMC bioinformatics. 2004;5:113.

10. Castresana J. Selection of conserved blocks from multiple alignments for their use in phylogenetic analysis. Mol Biol Evol. 2000;17:540-52.

11. Sassera D, Lo N, Epis S, D'Auria G, Montagna M, Comandatore F, et al. Phylogenomic evidence for the presence of a flagellum and cbb3 oxidase in the free-living mitochondrial ancestor. Mol Biol Evol. 2011;28(12):3285-96.

12. Abascal F, Zardoya R, Posada D. ProtTest: selection of best-fit models of protein evolution. Bioinformatics. 2005;21:2104-5.

13. Stamatakis A. RAxML version 8: a tool for phylogenetic analysis and post-analysis of large phylogenies. Bioinformatics. 2014;30:1312-3.

14. Badger JH, Eisen JA, Ward NL. Genomic analysis of Hyphomonas neptunium contradicts 165 rRNA gene-based phylogenetic analysis: implications for the taxonomy of the orders "Rhodobacterales" and Caulobacterales. Int I Syst Evol Microbiol. 2005;55:1021-6.

15. Gruber-Vodicka HR, Dirks U, Leisch N, Baranyi C, Stoecker K, Bulgheresi S, et al. Paracatenula, an ancient symbiosis between thiotrophic Alphaproteobacteria and catenulid flatworms. Proc Natl Acad Sci U S A. 2011;108:12078-83.

16. Eddy SRS. Profile hidden Markov models. Bioinformatics. 1998;14:755-63.

17. Li W, Godzik A. Cd-hit: a fast program for clustering and comparing large sets of protein or nucleotide sequences. Bioinformatics. 2006;22:1658-9.

18. Huson DH, Richter DC, Rausch C, Dezulian T, Franz M, Rupp R. Dendroscope: An interactive viewer for large phylogenetic trees. BMC Bioinformatics. 2007:8:460.

19. Retchless AC, Lawrence JG. Phylogenetic incongruence arising from fragmented speciation in enteric bacteria. Proc Natl Acad Sci U S A 2010;107:11453-8.

20. Hanage WP, Fraser C, Spratt BG. Fuzzy species among recombinogenic bacteria. BMC Biol. 2005;3:6

21. Bonaventura MPD, Lee EK, De Salle R, Planet PJ. A whole-genome phylogeny of the family Pasteurellaceae. Mol Phylogenet Evol. 2010;54:950-6.

22. Thrash JC, Pollock J, Torok T, Coates JD. Description of the nove perchlorate-reducing bacteria Dechlorobacter hydrogenophilus gen. nov., sp. nov. and Propionivibrio militaris, sp. nov. Appl Microbiol Biotechnol. 2010;86:335-43.

23. Coates JD, Michaelidou U, Bruce RA, O'Connor SM, Crespi JN, Achenbach LA. Ubiquity and diversity of dissimilatory (per)chlorate-reducing bacteria. Appl Environ Microbiol. 1999;65:5234-41.

24. Coates JD, Chakraborty R, Lack JG, O'Connor SM, Cole KA, Bender KS, et al. Anaerobic benzene oxidation coupled to nitrate reduction in pure culture by two strains of Dechloromonas. Nature. 2001;411:1039-43.

25. Bender KS, Shang C, Chakraborty R, Belchik SM, Coates JD, Achenbach LA. Identification, characterization, and classification of genes encoding perchlorate reductase. J Bacteriol. 2005;187:5090-6.

26. Simon J, Kern M. Quinone-reactive proteins devoid of haem b form widespread membrane-bound electron transport modules in bacterial respiration. Biochem Soc Trans. 2008;36:1011-6.

27. Kloer DP, Hagel C, Heider J, Schulz GE. Crystal structure of ethylbenzene dehydrogenase from Aromatoleum aromaticum. Structure. 2006;14:1377-88.

28. Clark IC, Melnyk RA, lavarone AT, Novichkov PS, Coates JD. Chlorate reduction in Shewanella algae ACDC is a recently acquired metabolism characterized by gene loss, suboptimal regulation and oxidative stress. Mol Microbiol. 2014;94:107-25.

29. Hofbauer S, Gruber C, Pirker KF, Sündermann A, Schaffner I, Jakopitsch C, et al. Transiently produced hypochlorite is responsible for the irreversible inhibition of chlorite dismutase. Biochemistry. 2014;53:3145-57.

30. Melnyk RA, Youngblut MD, Clark IC, Carlson HK, Wetmore KM, Price MN, et al. Novel mechanism for scavenging of hypochlorite involving a periplasmic methionine-rich peptide and methionine sulfoxide reductase. mBio. 2015;6(3):e00233-15.

31. Schwarz G, Mendel RR, Ribbe MW. Molybdenum cofactors, enzymes and pathways. Nature. 2009:460:839-47.

32. Rothery RA, Workun GJ, Weiner JH. The prokaryotic complex iron-sulfur molybdoenzyme family. Biochim Biophys Acta. 2008;1778:1897-929.

33. Hänzelmann P, Schindelin H. Binding of 5'-GTP to the C-terminal FeS cluster of the radical S-adenosylmethionine enzyme MoaA provides insights into its mechanism. Proc Natl Acad Sci U S A. 2006;103:6829-34.
34. Logan BE, Zhang H, Mulvaney P, Milner MG, Head IM, Unz RF. Kinetics of perchlorate- and chlorate-respiring bacteria. Appl Environ Microbiol. 2001;67:2499-506.

35. Domínguez NM, Hackett KT, Dillard JP. XerCD-mediated site-specific recombination leads to loss of the 57-kilobase gonococcal genetic island. J Bacteriol. 2011;193:377-88.

36. Carnoy C, Roten C-A. The dif/Xer recombination systems in proteobacteria. PLoS One. 2009;4:e6531.

37. Burrus V, Pavlovic G, Decaris B, Guédon G. Conjugative transposons: the tip of the iceberg. Mol Microbiol. 2002;46:601-10.

38. Clark IC, Melnyk RA, Engelbrektson A, Coates JD. Structure and evolution of chlorate reduction composite transposons. mBio. 2013;4:4.

39. Maixner F, Wagner M, Lücker S, Pelletier E, Schmitz-Esser S, Hace K, et al. Environmental genomics reveals a functional chlorite dismutase in the nitrite-oxidizing bacterium 'Candidatus Nitrospira defluvii'. Environ Microbiol. 2008;10:3043-56

40. Mlynek G, Sjöblom B, Kostan J, Füreder S, Maixner F, Gysel K, et al. Unexpected diversity of chlorite dismutases: a catalytically efficient dimeric enzyme from Nitrobacter winogradskyi. J Bacteriol. 2011;193:2408-17.

41. Yoshimatsu K, Sakurai T, Fujiwara T. Purification and characterization of dissimilatory nitrate reductase from a denitrifying halophilic archaeon, Haloarcula marismortui. FEBS Lett. 2000:470:216-20.

42. Martinez-Espinosa RM, Dridge EJ, Bonete MJ, Butt JN, Butler CS, Sargent F, et al. Look on the positive side! The orientation, identification and bioenergetics of "Archaeal" membrane-bound nitrate reductases. FEMS Microbiol Lett. 2007;276:129-39.

43. Van Ginkel SW, Lamendella R, Kovacik WP, Santo Domingo JW, Rittmann BE. Microbial community structure during nitrate and perchlorate reduction in ion-exchange brine using the hydrogen-based membrane biofilm reactor (MBfR). Bioresource Technol. 2010;101:3747-50.

44. Xiao Y, Roberts DJ, Zuo G, Badruzzaman M, Lehman GS. Characterization of microbial populations in pilot-scale fluidized-bed reactors treating perchlorate- and nitrate-laden brine. Wat Res. 2010:44:4029-36.

45. Kounaves SP, Stroble ST, Anderson RM, Moore Q, Catling DC, Douglas S, et al. Discovery of natural perchlorate in the Antarctic Dry Valleys and its global implications. Environ Sci Technol. 2010;44:2360-4.

46. Kuo $\mathrm{C}-\mathrm{H}$, Ochman $\mathrm{H}$. The extinction dynamics of bacterial pseudogenes. PLoS Genet. 2010;6:8

47. Liu Y, Harrison PM, Kunin V, Gerstein M. Comprehensive analysis of pseudogenes in prokaryotes: widespread gene decay and failure of putative horizontally transferred genes. Genome Biol. 2004;5:R64

\section{Submit your next manuscript to BioMed Central and take full advantage of:}

- Convenient online submission

- Thorough peer review

- No space constraints or color figure charges

- Immediate publication on acceptance

- Inclusion in PubMed, CAS, Scopus and Google Scholar

- Research which is freely available for redistribution 